\title{
Linx
}

Revue des linguistes de l'université Paris X Nanterre

$57 \mid 2007$

Études de syntaxe : français parlé, français hors de

France, créoles

\section{Pour une approche comparative des dynamiques structurelles du français en Afrique}

\section{Katja Ploog}

\section{(2) OpenEdition}

\section{Journals}

Édition électronique

URL : http://journals.openedition.org/linx/307

DOI : $10.4000 /$ linx.307

ISSN : 2118-9692

Éditeur

Presses universitaires de Paris Nanterre

Édition imprimée

Date de publication : 1 décembre 2007

Pagination : 165-176

ISSN : 0246-8743

Référence électronique

Katja Ploog, "Pour une approche comparative des dynamiques structurelles du français en Afrique », Linx [En ligne], 57 | 2007, mis en ligne le 15 février 2011, consulté le 15 mai 2020. URL : http:// journals.openedition.org/linx/307 ; DOI : https://doi.org/10.4000/linx.307 


\title{
Pour une approche comparative des dynamiques structurelles du français en Afrique
}

\author{
Katja Ploog \\ Université de Franche-Comté et Université de Fribourg
}

Dans son usage par les populations africaines, le français subit des restructurations. En Côte d'Ivoire par exemple, la dynamique sociale de l'appropriation du français l'a fait passer en moins d'un siècle du supervéhiculaire très minoritaire à la langue urbaine par excellence ; la dynamique structurelle a abouti à des usages locaux suffisamment divergents $\mathrm{du}$ français standard ${ }^{1}$ pour mettre en péril l'intercompréhension avec la francophonie extérieure.

Nous entendons par dynamique langagière l'interprétation de séquences discursives, structurellement sous-déterminées, par un auditeur qui les catégorise en vue d'un réinvestissement. La dynamique langagière est ainsi constitutive de toute activité discursive, de production et d'écoute, avec pour fait élémentaire le non-standard ${ }^{2}$; il n’y a que «légiférer» sur les usages qui puisse les soustraire à la dynamique constitutive des codes communautaires (... des « langues »). Afin de contribuer à une approche empirique de ces dynamiques, nous esquissons ici une étude comparée de pratiques linguistiques en francophonie.

Si les restructurations peuvent en dernier lieu être interprétées comme «effet secondaire » de l'immiscion du français dans une écologie linguistique, le premier

\footnotetext{
${ }^{1}$ Le terme standard sera utilisé ici au sens de la norme grammaticale, explicite, prescriptive, prestigieuse.

${ }^{2}$ Nous n'entrerons pas ici dans le débat de savoir s'il y a une terminologie plus adéquate en raison de sa primauté.
} 
objectif consistera à dégager les facteurs socio-démographiques qu'elles sous-tendent. Faute de données comparatives fiables, nous proposons quelques hypothèses relatives aux restructurations possibles et probables dans les constellations sociales de la francophonie africaine.

\section{L'émergence des restructurations et leur description}

Pour Hopper (1987:142), les constructions linguistiques émergent du discours dans un processus constant, et il n'y a pas lieu de postuler une différence qualitative entre la routinisation de stratégies constructionnelles et des régularités plus figées; autrement dit, il n'y a pas de grammaire a priori. Nous en déduisons que le non-standard joue un rôle central dans les restructurations.

Pour reprendre ce postulat, nous allons d'abord recourir à la notion d'élaboration, en gardant du concept de Kloss (1967) la démarche de conventionnalisation, mais en l'attribuant au locuteur-auditeur dans son activité discursive habituelle. La complexité de la tâche d'élaboration du discours est fonction du réseau de communication dans lequel elle s'accomplit : elle est déterminée par les facteurs linguistiques définitoires du contexte interactionnel. Plus la constellation sociale est instable, plus les refontes des modalités de communication sont nombreuses; plus une communauté est hétérogène, plus les dynamiques langagières y sont marquées. Thomason (2000) affirme toutefois qu'il n'existe pas de contrainte linguistique à proprement parler (condition nécessaire) régissant les influences potentielles entre les langues présentes dans un espace communicatif, et qu'aucun cadre social (condition suffisante) ne laisse prévoir le changement linguistique. Reste que la description de la dynamique doit être fondée sur une connaissance détaillée des procès sociaux et de l'histoire sociodémographique d'une communauté ${ }^{3}$, ce qui sera l'objet de la section 3 .

Ensuite, pour décrire une élaboration divergente de la norme établie en termes de restructuration, nous postulerons que celle-ci représente la sédimentation de nouvelles routines dans un cadre discursif dont les mécanismes sont destinés à maitriser les tensions entre différentes exigences pragmatico-sociales auxquelles un locuteur doit faire face, liées à la gestion de la référentialité (comme la valeur + [animé] pour la construction d'un référent nominal), de la linéarité (comme les contraintes de réduction de clitiques) et de l'interlocutivité - immédiate et permanente - qui détermine des variations par exemple en fonction de la situation de communication plus ou moins formelle.

Ceci conduit à délimiter comme observable non pas une unité formelle ou fonctionnelle, mais un microsystème, en tant que faisceau complexe de formes et de fonctions entretenant des liens privilégiés. Par conséquent, la délimitation d'un microsystème peut (doit) varier d'une étude à une autre ; une forme peut être attribuée à plusieurs microsystèmes. Par exemple, en français standard, le microsystème des constituants clitiques des objets verbaux s'organise sur deux positions syntaxiques dominées par l'opposition \pm [direct] ; des contraintes supplémentaires de linéarisation (p.ex. de personne) régissent la cooccurrence de deux éléments. La forme LUI, qui

${ }^{3}$ La question de savoir s'il existe des processus spécifiques au contact de langues ne sera pas traitée ici. 
appartient à ce microsystème, peut être étudiée par ailleurs dans le microsystème du premier actant ou dans celui des pronoms personnels; ces entrées participent aussi du microsystème des compléments verbaux. Si la focalisation de phénomènes 'locaux' dans l'observation de dynamiques globales est une nécessité pratique, le microsystème parait être le plus petit ensemble susceptible de prendre en compte l'hétérogénéité des pratiques. La section 4 sera consacrée à la discussion des microsystèmes dans un cadre comparatif.

Lorsqu'une caractéristique non standard se stabilise comme unité d'un microsystème, on parlera de restructuration, ou modification des stratégies constructionnelles. La restructuration peut concerner des traits fonctionnels ou formels (Heine \& Kuteva 2005). Pour les clitiques objet, on note par exemple dans l'usage abidjanais l'émergence du trait \pm [animé] comme pertinent: les actants à référent -[animé] ne sont pas élaborés dans la zone clitique. Un constituant animé en objet semble pouvoir être exprimé par LUI ou par LE, mais l'opposition \pm [direct] est neutralisée. Le poids de la restructuration est déterminé par sa routinisation dans les discours sociaux et représente une réorganisation partielle des normes. L'alternance LUI/LE tend à s'organiser autour du trait \pm [formel] : on observe la récurrence de LE (sous-représentation de LUI) en contexte informel et celle de LUI (sousreprésentation de LE) en contexte formel :

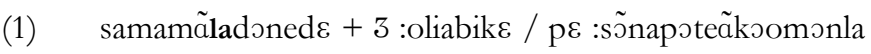

3+POSS-maman+SJ 3+SG+OBJ-SG+perf-donner PL-joli.habit COMP personne+SJ (NEG) SG+PERF-porter encore LOC-monde-LA ${ }^{4}$

Sa maman lui a donné des habits plus jolis que personne au monde n'en avait jamais porté (Ploog ABJ97, B46Y :IV.10/004)

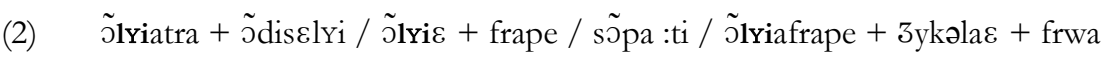

3+SJ-3+SG+OBJ-attraper-PRES 3+SJ-SG+PRES+dire 3+N+SJ-SG+être $3+$ SG 3+SJ-3+SG+OBJ-SG+PERF-frapper [Ø+SJ] PL+PERF-partir 3+SJ-3+SG+OBJ-SG+PERF-frapper jusqu'à-être+PRES-froid

On l'attrape, on dit c'est lui. On l'a frappé. «Ils ont continué ». On l'a frappé jusqu'à ce qu'il fasse froid. (Ploog ABJ97, GM2Y :III.05/026)

L'opposition selon le trait \pm [formel] ajouté à \pm [animé] est interprétée comme normalisation de nouvelles caractéristiques du microsystème, qui se trouve enrichi puisqu'il s'ajoute à l'opposition standard \pm [direct], en entrant en collision partielle avec celle-ci. Une conséquence méthodologique est la place centrale des ambiguités structurelles, notamment par l'étude des zones environnantes, incluant d'autres microsystèmes comme les structures prédicatives des verbes, les positions syntaxiques du constituant sujet, le complémenteur, etc.

\footnotetext{
${ }^{4}$ Transposition morphologique des exemples transcrits : majuscules pour les morphèmes fonctionnels, trait pour les frontières morphémiques, plus pour un assemblage de plusieurs valeurs «dans» un morphème, point pour la liaison des compositions lexicales.
} 


\section{Hétérogénéité discursive et hétérogénéité sociale : corpus}

\subsection{Phases historiques de la dynamique linguistique abidjanaise}

Pour esquisser la dynamique sociale ivoirienne supposée conditionner la dynamique linguistique, en l'absence de données statistiques précises, de documentation linguistique et de corpus contrôlés avant 1960, les énoncés sur formes et fonctions du français jusqu'à cette période restent des hypothèses :

(1) Installation du pouvoir colonial (1893-1933): premiers contacts en zone côtière (français / langues kwa) suite à l'ouverture d'écoles missionaires. Les fonctions du français étaient alors véhiculaires et ultra-minoritaires (liées au culte et à l'administration).

(2) Expansion urbaine (1933-1951): le siège du gouverneur est transféré à Abidjan, ce qui entraîne le développement urbain; intensification des contacts ( $\sim 30$ ethnies : groupes kwa/diula/français). L'expression en français augmente quantitativement et qualitativement, la fonction de langue seconde se développe. Les mécanismes de code-switching (communicatif/palliatif) et les alternances ont pu être caractéristiques.

(3) Le miracle ivoirien (1951-1975): dans un contexte de forte compétition sociale, une population linguistiquement très hétérogène plébiscite le français comme langue de communication interethnique et fait émerger le 'français populaire d'Abidjan' (FPA). L'explosion démographique accentue les carences du système éducatif et le désir d'intégration des masses populaires. C'est dans cette phase que la réplication du trait \pm [animé] dans la zone clitique a pu avoir lieu.

(4) Le mirage ivoirien (1975-1985): les perdants de la compétition urbaine revendiquent une identité par la création de modalités discursives spécifiques (le 'nouchi') à partir de sources structurelles autochtones, dont le FPA est la pièce majeure. La scolarisation ne progresse pas et le désenchantement gagne du terrain - l'adaptation est contre-carrée par un mouvement de ségrégation. Si le code-switching communicatif a quasiment disparu, le code-switching identitaire se ritualise dans les domaines caractéristiques de l'argot.

(5) Emergence de l'ivoirité (1985-1997) : les pratiques se diversifient tout en restant focalisées sur le français: le FPA est transmis à la génération suivante et évolue structurellement; le nouchi est utilisé dans des courants artistiques émergents et fait l'objet de réappropriation; la diffusion du français standard ne progresse pas. L'émergence du trait \pm [formel] se situerait ici : les formes LUI qui ne correspondent pas à un objet indirect constituent un ajustement vers le standard du LE + [humain] non standard, qui s'avère erroné lorsque LE représente un objet direct. 
(6) Consolidation de l'ivoirité (<1997): le nouchi se généralise. La familiarité passive est l'aspect le plus répandu du plurilinguisme à Abidjan. Tous les locuteurs urbains grandissent avec le français, et le plurilinguisme individuel diminue rapidement.

\subsection{Facteurs sociodémographiques des dynamiques langagières}

Le fait démographique majeur de la dynamique abidjanaise est la pression sociale : croissance urbaine intense, forte hétérogénéité des populations impliquées, densité des réseaux sociaux. Ces vecteurs, décrits par la sociologie urbaine de l'Ecole de Chicago $^{5}$, peuvent être reformulés comme facteurs de l'écologie des dynamiques langagières (cf. aussi Mufwene 2001 :136) :

- Le facteur nombre définit la stabilité de la situation linguistique : le nombre de langues ou de communautés d'origine au pro rata de la population définit l'éventail de matériaux de construction disponibles; leur représentation dans les domaines communicationnels et le nombre de locuteurs par langue (et les plurilinguismes individuels) permettent d'identifier les langues les plus exposées aux restructurations.

- L'bétérogénéité - de familles et de types linguistiques - définit la diversité et les atouts pragmatiques respectifs des matériaux disponibles.

- La densité de réseaux de communication définit les paramètres du contact entre modèles/matériaux différents. On recensera sous ce chapeau les caractéristiques sociologiques qui déterminent les interactions dans la communauté (taux d'alphabétisation élevé, forte urbanisation etc.).

Enfin, il faut tenir compte de la variabilité des facteurs au cours des différentes phases.

\subsection{Constellations sociales, dynamiques langagières}

L'annexe présente les caractéristiques de 5 états francophones: Guinée, Sénégal, Cameroun, Gabon, outre la Côte d'Ivoire, base de la grille.

La Guinée est un des rares pays d'Afrique francophone qui a, pendant plus de 25 ans (sous Sékou Touré, de 1958 à 1984), appliqué une politique en faveur des langues ethniques, le pays étant coupé des réseaux occidentaux et francophones. Le français assume surtout des fonctions véhiculaires, en restant réservé aux populations urbaines scolarisées. On peut rapprocher cette situation de la phase (2) de la dynamique ivoirienne, et faire l'hypothèse que les mécanismes d'acquisition d'une langue seconde (code-switching communicatif et alternance) prévalent : les processus engagés seraient de l'ordre du copiage de traits des langues maternelles, dont l'influence est d'autant plus plausible qu'elles sont peu nombreuses, que le zonage de l'espace urbain a produit des quartiers relativement homogènes, et que les dynamiques individuelles peuvent ainsi être supposées convergentes. Certaines de ces langues, promues par le

${ }^{5}$ Wirth (1938) in Grafmeyer \& Joseph (1984). 
système scolaire jusqu'en 1984, relèvent du même groupe qu'en Côte d'Ivoire (mande). $\mathrm{Si}$ la situation guinéenne est actuellement relativement stable (peu ou pas d'immigration, croissance démographique faible), la dynamique restera faiblement marquée.

Le Sénégal manifeste une dynamique assez différente de celle de la Côte d'Ivoire, alors que les composantes de l'écologie paraissent similaires : face aux langues vernaculaires, les véhiculaires wolof et français, tous deux associés à l'urbanité, sont spécialisés respectivement à l'oral et à l'écrit. A l'avenir, le bilinguisme (ou plurilinguisme) coordonné individuel - de fait déjà très développé - conduirait à l'affirmation de l'identité urbaine lettrée par la valorisation du code-switching (socio-indexical). Si la faible scolarisation et le bilinguisme collectif perdure, on peut s'attendre à des restructurations mineures des microsystèmes en faveur d'une convergence entre français et wolof.

Le Cameroun est probablement la commuanuté nationale la plus hétérogène du sous-continent. La politique linguistique ignore les langues autochtones, qui continuent toutefois à assurer les fonctions véhiculaires régionales, ce qui a d'autant plus de poids que le pays possède une densité communicationnelle faible. Dans les centres urbains, des codes mixtes ont émergé (Pidgin English depuis le siècle dernier, plus récemment camfranglais). Avec un taux d'alphabétisation moyen (élevé comparé au Sénégal ou à la Guinée), ce profil plurilingue et décentralisé conduira à des pratiques diverses du français, qui peut prendre la forme d'un bilinguisme collectif à la guinéenne dans le Nord et à la sénégalaise dans certains centres urbains, jusqu'à, localement (Yaounde), une diversification interne à métissage variable, de l'ordre de la phase ivoirienne (4).

Le Gabon présente un morcellement linguistique tel que le français s'est imposé comme langue véhiculaire (et officielle) sans conteste; il semble en passe de devenir première langue maternelle, au moins en milieu urbain. Il y a donc des similitudes avec la Côte d'Ivoire (nombre et hétérogénéité), bien que la densité communicationnelle favorise la diffusion du standard. Néanmoins, la forte adhésion de la communauté au français favorise les mécanismes de ségrégation/sous-catégorisation sociale et l'apparition de modalités discursives identitaires avec pour base principale les variantes non standard du français. Pour les langues ethniques, on peut s'attendre à une attrition progressive, puis au shift, comme en Côte d'Ivoire depuis la phase (5).

Aucune des constellations ne semble actuellement propice à une pidginisation du français, processus d'appropriation massive en Côte d'Ivoire qui a donné lieu au FPA (phase 3).

\section{Hétérogénéité discursive et hétérogénéité structurelle}

\subsection{Constellations originales et comparaison}

Notre hypothèse est que le français a été adapté aux exigences pragmatiques des constellations présentées. La révision a pour effet la reconduction ou la restructuration des microsystèmes existants. Nous proposerons dans la suite quelques pronostics sur les caractérististiques structurelles, au vu des configurations discursives pertinentes dans la constellation sociolinguistique. Notons que le contact de langues est un aspect de l'hétérogénéité sociale qui préfigure, à travers le répertoire composite du locuteur, le 
résultat formel d'une restructuration donnée. L'établissement même de transferts structurels restant problématique, il conviendra d'accorder une grande importance à cette dimension.

\subsection{Observables morphosyntaxiques}

Suite à l'analyse du corpus Ploog ABJ97, nous avons retenu comme observables quatre microsystèmes sujets à restructuration: les clitiques objet de $3^{\text {e }}$ personne $\left(\mathrm{LE} / \mathrm{LUI}^{6}\right)$; la complémentation verbale et nominale (QUE) ; l'élaboration formelle de la fonction sujet (double-marquage, PRO, Ø); les fonctions sémanticopragmatiques de l'enclitique LA.

Sur le plan méthodologique, les restructurations fonctionnelles ne seront dans un premier temps détectées que dans les élaborations déviant du standard (les traits fonctionnels ne sont pas visibles/audibles a priori). Les observables semblent néanmoins présenter un intérêt majeur au-delà de la Côte d'Ivoire, ce qui devrait faciliter le paramétrage des données et assurer l'interprétabilité structurelle - pour résister à la tentation d'explication intersystémique hâtive. Les deux derniers observables ne seront pas développés ici, malgré leur pertinence, liée à une forte imbrication des microsystèmes.

Nous ne reviendrons pas sur le premier observable, les clitiques objet, présenté dans la section 2. Le second observable, la complémentation syntaxique verbale et nominale, dont le centre forme le constituant que, participe, en français standard, d'un paradigme d'oppositions fourni pour les expansions nominales. En français parlé, ces oppositions semblent en partie neutralisées au profit du seul que, et alternent pour les compléments verbaux avec d'autres marques $(\varnothing$, marqueurs prosodiques, marqueurs lexicaux) dans un microsystème de rection verbale qui a pu être qualifié de faible (Blanche-Benveniste 1989). Les raisons du choix de cet observable sont analogues à celles du précédent.

\subsection{Données comparatives : bypothèses}

\subsection{1. $L E-L U I$}

Sur le plan cognitif, les constituants animés sont des participants au procès verbal plus prototypiques et semblent universellement privilégiés pour une élaboration formelle (phonétique, morphologique). Une réorganisation de la zone clitique qui valorise le trait \pm [animé] est ainsi susceptible de se produire dans toutes les constellations. La neutralisation du trait \pm [direct] s'inscrit dans la même dynamique, qui donne priorité au pragmatique sur le syntaxique. La préférence pour le paradigme LE en non-standard peut s'expliquer par le processus d'analogie (ou principe de congruence) : la $3^{e}$ personne se régularise sur les deux premières (me / te / le).

Les deux restructurations de traits fonctionnels et la préférence pour LE doivent connaitre la 'résistance' la plus forte là où le modèle scolaire est le plus présent, au Gabon et au Sénégal. Le corpus guinéen semble montrer d'ores et déjà une réorganisation plus large du microsystème des arguments verbaux, où la distinction des

${ }^{6}$ Les majuscules indiquent qu'il s'agit des lemmes. 
fonctions syntaxiques \pm [direct] semble en voie de neutralisation même au niveau nominal :

(3) le policie a demade le commerçant le comemerçant a spique tousequipase (Laseldi/AUF CON03, D246)

Par cette perte de sensibilité grammaticale, le corpus de Conakry diffère de l'abidjanais, où la marque indirecte à est généralement maintenue; on peut y voir l'expression immédiate de la scolarisation, en l'absence d'un usage social généralisé.

L'alternance de LE et LUI en fonction de la configuration interactionnelle (trait \pm [formel]) relève du mécanisme d'accommodation qui pourra gagner d'autant plus de représentativité que la diglossie sera conflictuelle. Dans une certaine mesure, on peut se demander si l'émergence de cette opposition dans le microsystème n'est pas rendue possible suite à la valorisation du trait \pm [animé]. Il est alors plausible qu'il émerge dans les constellations où le français possède des fonctions sociales véritables, mais où la diffusion de la norme reste limitée; son élaboration à terme semble donc plus probable en Guinée qu'au Gabon.

\subsection{2. $Q U E$}

Le complémenteur QUE possède à la fois une fonction de segmentation et de cohésion entre deux ensembles propositionnels et/ou énonciatifs; nous nous en tiendrons ici aux caractéristiques de la complémentation verbale.

Italia (2005 :203) montre que l'expansion verbale - où le complémenteur indique le discours rapporté - se fait à l'oral plus fréquemment par une pause ou une balise discursive (bon, oh eh, ah) qu'au moyen du QUE, la plupart étant en outre doublés d'une balise :

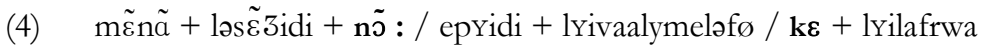

maintenant le singe il dit non [pause] et puis il dit [changement mélodique] lui (il) va allumer le feu que [pause] lui il a froid (Ploog ABJ97, B46Y :IV.10.40)

Dans cet exemple, la transposition d'une personne du discours en tierce personne est réalisée suivant les principes du discours indirect, alors que les exemples donnés par Italia de balises non standard concernent davantage des rapports de parole directs. A travers un corpus d'apprenants FLS de différents niveaux, l'auteure dégage trois types d'indices de marquage du discours rapporté, qu'elle place dans l'ordre d'acquisition suivant : marque prosodique (pause) $>$ lexicale (balise) $>$ grammaticale (complémenteur). L'approche quantitative semble corroborer l'hypothèse que le marquage (oral) par QUE sert la coordination d'unités propositionnelles sur le même plan énonciatif, comme en témoigne la troisième occurrence de l'exemple (4). Dans le corpus guinéen, QUE semble exprimer soit la dépendance d'une proposition antérieure, soit une finalité (pour que) ou une injonction :

(5) C'est ma mère qui ma commissione chez le commerçant que je ve acheter le sucre (Laseldi/AUF CON03, D287) 
(6) il me venir de de - demander que - est-ce que c'est bon est-ce que ce n'est pas bon (basilectal, Italia GAB05, cité dans Italia 2005 :202, B2)

On peut évidemment postuler l'insensibilité à l'une ou à l'autre des contraintes (enchâssement, décrochement) en situation de langue seconde. Mais si le décrochage énonciatif par une pause et l'emploi d'une marque lexicale/syntaxique de connexion en contexte ambigu sont caractéristiques d'un emploi oral, seule l'absence remarquable du complémenteur reflèterait alors un stade d'acquisition intermédiaire du français standard, là où le répertoire est trop succinct pour permettre au locuteur d'évaluer de manière appropriée l'exigence de marquage des décrochements énonciatifs. La présence remarquable de QUE correspond alors à deux dynamiques divergentes (Côte d'Ivoire vs. Guinée).

On doit donc s'attendre à ce que les balises non standard s'affirment dans les constellations où l'usage oral est suffisamment important pour avoir donné lieu à des traditions discursives spécifiques (Gabon, Cameroun). Les formes de balises non standard seront particulièrement conditionnées par la diversité des modèles linguistiques sous-jacents, du français standard aux langues locales : la position étant syntaxiquement peu contrainte ${ }^{7}$, son élaboration sera d'autant plus aisément marquée par des paramètres socio-indexicaux. Là où l'usage du français reste dominé par l'environnement institutionnel (Guinée, Sénégal), on peut postuler que l'acquisition guidée focalise sur l'élément le plus central et mène à la surgénéralisation de que, stratégie qui peut être partagée par les autres constellations (mais qui y montrera moins d'effets globaux).

\section{Bilan}

La vérification de ces hypothèses repose sur la constitution de corpus contrôlés, permettant de documenter les dynamiques par le recueil des interactions qui la promeuvent : par exemple, la cohésion du récit repose sur des stratégies de structuration dominées par les exigences pragmatiques caractéristiques de l'oral (prédominance de la relation interlocutive et de la linéarité du discours).

Or, les données anciennes sont écrites : correspondances coloniales ou projections humoristiques (caricaturales) des phénomènes dans les médias locaux. Les données récentes publiées - dont nous nous inspirons en attendant - sont souvent des inventaires dont l'origine est piètrement documentée. Par ailleurs, le cadre interactionnel habituel des recueils ('interview) laisse trop d'espace à une élaboration structurelle dominée par la vigilance normative ; certains aspects du microsystème ne seront peut-être jamais représentés, alors qu'ils existent, et parfois de longue date.

La plupart des recueils disponibles peuvent ainsi être qualifiés de "stéréotypants», non contrôlés. La conséquence est deux exigences méthodologiques pour l'établissement des dynamiques langagières: maittriser l'articulation de l'hétérogénéité d'un microsystème par un choix approprié des situations d'enquête; et situer les caractéristiques observées par des études quantitatives et longitudinales dans les

${ }^{7}$ Cependant, il doit s'agir d'un élément relativement court, le plus souvent monosyllabique. 
situations de communication appropriées. Concernant les contacts de langue, établir leur influence requiert, outre une description structurelle fiable de la langue source, une approche diachronique de la langue répliquée en comparant ses caractéristiques avant et après les processus de transfert présumés.

\section{RÉFÉRENCES BIBLIOGRAPHIQUES}

BiLOA, E. (2003), La langue française au Cameroun. Analyse linguistique et didactique. Peter Lang, Bern.

Blanche-Benveniste, C. (1989), "Constructions verbales 'en incise' et rection faible des verbes ». Recherches sur le français parlé 9, 53-73.

Calvet, L.-J. (1999), Pour une écologie des langues du monde. Plon, Paris.

DAfF, M. (1998), «Petite vitrine syntaxique du français oral en Afrique». In Queffelec, Ambroise (ed.), 1998, Alternances codiques et français parlé en Afrique. Publications de l'Université de Provence, Aix-en-Provence, 107-129.

Grafmeyer, Y. \& I. JosePh (eds.) (1984), L'école de Chicago. Naissance de l'écologie urbaine. Aubier, Paris.

Heine, B. \& T. Kuteva (2005), Language contact and Grammatical Change. Cambridge University Press, Cambridge.

Hopper, P.J. (1987), « Emergent Grammar ». Berkeley Linguistics Society, vol. 13, 139-157.

ITALiA, M. (2005), «Variations en fonction de l'âge et du degré de scolarité dans le français oral de locuteurs gabonais : la question du discours rapporté ». In : Ploog K. \& B. Rui (éds.), Appropriations du français en contexte multilingue. Eléments sociolinguistiques pour une réflexion didactique à propos de situations africaines. PUFC, Besançon, 199-216.

(2006), «Le morphème là dans les variétés mésolectales et basilectales en français du Gabon ». Le français en Afrique (ROFCAN) 21, 281-290.

KLoss, H. (1967), «Abstand-languages and Ausbau-languages ». Anthropological linguistics 9 (rediffusion in: A Retrospective of the Journal Anthropological Linguistics : Selected Papers, 1959-1985 = AL 35/1993, 158-170).

LANGaCKer, R.W. (1977), «Syntactic Reanalysis ». In: Li Ch. (ed.), Mecanisms of syntactic change. University of Texas Press, Austin, 57-139.

LeHmann, Ch. (2002) (1995), Thoughts on Grammaticalization. (2nd revised edition) Lincom Europa, Munich.

Mufwene, S.S. (2001), The Ecology of Language Evolution. Cambridge University Press, Cambridge.

Thomason, S.G. (2001), Language contact: an introduction. Edinburgh University Press, Edinburgh.

(2000), « On the unpredictability of contact effects ». Estudios de Sociolinguistica 1.1, 173182. 
Pour une approche comparative des dynamiques structurelles du français en Afrique

\section{CORPUS}

[Italia GAB05] : Corpus oral recueilli auprès de locuteurs fang peu ou pas lettrés dans le Woleu Ntem (Nord du Gabon).

[Laseldi/AUF CON03] : Corpus de 400 narrations écrites et 8,5h d'interviews et de narrations orales recueilli en 2003 à Conakry (Guinée) avec des écoliers de 5e année dans deux écoles primaires.

[Ploog ABJ97] : Corpus de 23h d'interactions spontanées, d'interviews et de narrations orales recueilli en 1997 à Abidjan (enfants de la rue, écoliers de quartiers populaires, travailleurs de l'économie populaire spontanée). 


\section{Annexe : Caractéristiques socio-démographiques des Etats ${ }^{8}$}

\begin{tabular}{|c|c|c|c|c|c|}
\hline & $\begin{array}{c}\text { CÔTE } \\
\text { D'IVOIRE }\end{array}$ & GUINÉE & SÉNÉGAL & CAMEROUN & GABON \\
\hline Habitants (1) & 18,1 mill & 9,4 mill & 11,6 mill & 17,8 mill & 1,3 mill \\
\hline $\begin{array}{l}\text { Groupes } \\
\text { ethniques (4) }\end{array}$ & 78 & 34 & 36 & 282 & 41 \\
\hline $\begin{array}{l}\text { Langues } \\
\text { véhiculaires }\end{array}$ & $\begin{array}{c}\text { Français } \\
(+ \text { diula, } \\
\text { anyi/baule })\end{array}$ & $\begin{array}{c}\text { Susu } \\
\text { (urbain) }\end{array}$ & $\begin{array}{l}\text { Wolof (urbain), } \\
\text { français (lettré) }\end{array}$ & $\begin{array}{l}\text { (régionaux :) fulfulde, } \\
\text { beti, basaa, bulu, } \\
\text { ewondo, douala } \\
\text { français, pidgin } \\
\text { english, ?camfranglais }\end{array}$ & Français \\
\hline $\begin{array}{l}\text { Croissance } \\
\text { démogra- } \\
\text { phique (1) }\end{array}$ & $\begin{array}{c}3,4 \% \\
(15 \% \text { vers } 1960)\end{array}$ & $2,2 \%$ & $2,4 \%$ & $1,8 \%$ & 1,4 \\
\hline $\begin{array}{l}\text { Familles } \\
\text { linguistiques } \\
(4)\end{array}$ & $\begin{array}{c}4 \text { (kru, gur, } \\
\text { kwa, mande) }+ \\
\text { immigrés }\end{array}$ & $\begin{array}{l}2 \text { (mande, } \\
\text { ouest- } \\
\text { atlantique) }\end{array}$ & $\begin{array}{c}1 \text { (ouest- } \\
\text { atlantique) }\end{array}$ & $\begin{array}{l}3 \text { (bantu, bantoïde- } \\
\text { sud, adamawa) }\end{array}$ & 1 (bantu) \\
\hline $\begin{array}{l}\text { Morcellemen } \\
\mathrm{t}(4)\end{array}$ & $\begin{array}{c}17 \text { langues } \\
\text { pour } 45 \% \text { de la } \\
\text { population }\end{array}$ & $\begin{array}{l}6 \text { langues } \\
\text { pour } 60 \%\end{array}$ & $\begin{array}{c}5 \text { langues pour } \\
80 \% \\
\text { (wolof : } 42 \% \text { ) } \\
\end{array}$ & 23 langues pour $40 \%$ & $\begin{array}{l}13 \text { langues } \\
\text { pour } 35 \%\end{array}$ \\
\hline $\begin{array}{l}\text { Langues } \\
\text { standardisées } \\
\text { (3) }\end{array}$ & Français & $\begin{array}{c}\text { Français } \\
\text { (avant } \\
1984: \\
\text { susu, } \\
\text { maninka, } \\
\text { pulaar) }\end{array}$ & $\begin{array}{c}\text { Trilingue: } \\
\text { alphabétisation } \\
\text { première en } \\
\text { LM, } \\
\text { enseignement } \\
\text { du wolof puis } \\
\text { du/en français }\end{array}$ & $\begin{array}{c}\text { Français, anglais } \\
\text { (territorialisé) }\end{array}$ & Français \\
\hline $\begin{array}{l}\text { Langues de } \\
\text { scolarisation } \\
\text { (3) }\end{array}$ & $\begin{array}{c}\text { Français } \\
\text { (normalisation } \\
\text { patielle : bété, } \\
\text { baule, diula, } \\
\text { senoufo) }\end{array}$ & $\begin{array}{l}\text { Français, } \\
\text { susu, } \\
\text { maninka, } \\
\text { pulaar }\end{array}$ & $\begin{array}{l}\text { Français, wolof, } \\
\text { puular, serer, } \\
\text { diola, malinke, } \\
\text { soninke (etc) }\end{array}$ & Français, anglais & Français \\
\hline $\begin{array}{l}\text { Densité de la } \\
\text { population } \\
\text { (1) }\end{array}$ & $49,7 \mathrm{hab} / \mathrm{km} 2$ & $\begin{array}{c}37 \\
\mathrm{hab} / \mathrm{km} 2\end{array}$ & $52 \mathrm{hab} / \mathrm{km} 2$ & $31 \mathrm{hab} / \mathrm{km} 2$ & $\begin{array}{c}4,6 \\
\mathrm{hab} / \mathrm{km} 2\end{array}$ \\
\hline $\begin{array}{l}\text { Urbanisation } \\
\text { (1) }\end{array}$ & $45 \%$ & $29,6 \%$ & $50 \%$ & $>50 \%$ & $80 \%$ \\
\hline $\begin{array}{l}\text { Communciat } \\
\text { ion } \\
\text { publique : } \\
\text { religions, } \\
\text { médias }(3 / 1)\end{array}$ & $\begin{array}{c}\text { 1) islam } 38 \% \text {, } \\
\text { christianisme } \\
22 \%, \\
\text { animisme } 17 \% \\
\text { 2) français } 9\end{array}$ & $\begin{array}{l}\text { 1) islam } \\
85 \% \\
\text { 2) français, } \\
\text { susu, } \\
\text { maninka, } \\
\text { pulaar }\end{array}$ & $\begin{array}{l}\text { 1) islam } 94 \% \\
\text { 2) écrits : } \\
\text { français; oraux : } \\
\text { wolof }\end{array}$ & $\begin{array}{l}\text { 1) christianisme } \\
>35 \% \text {, islam }>15 \% \text {, } \\
\text { animisme }>45 \% \\
\text { 2) français, anglais }{ }^{12}\end{array}$ & $\begin{array}{c}\text { 1) } \\
\text { christianis } \\
\text { me } 90 \% \\
\text { 2) } \\
\text { Français }^{12}\end{array}$ \\
\hline $\begin{array}{l}\text { taux } \\
\text { d'alphabétisa } \\
\text { tion }(2 / 1)\end{array}$ & $25 \% / 48,7 \%$ & $\begin{array}{c}25,4 \% / \\
29,5 \%\end{array}$ & $35 \% / 39 \%$ & $67,9 \%$ & $80 \%$ \\
\hline $\begin{array}{l}\text { structure } \\
\text { sociale } \\
\text { (représentée } \\
\text { ici par le } \\
\text { PIB) (1) }\end{array}$ & $866 \$ /$ hab & $332 \$ /$ hab & $710 \$ /$ hab & $?$ & $?$ \\
\hline
\end{tabular}

${ }^{8}$ Sources : (1) http ://www.diplomatie.gouv; (2) http ://www.stat.-guinee.org ;

(3) http://tlfq.ulaval.ca/axl/afrique/; (4) http://www.ethnologue.com, les données présentées dans le tableau sont partiellement calculées par l'auteure à partir des données du site.

${ }^{9}$ La diffusion de programmes radio en langues nationales est très marginale. 